\title{
A POSTERIORI ERROR ESTIMATES BY RECOVERED GRADIENTS IN PARABOLIC FINITE ELEMENT EQUATIONS
}

\author{
D. LEYKEKHMAN AND L. B. WAHLBIN
}

\begin{abstract}
This paper considers a posteriori error estimates by averaged gradients in second order parabolic problems. Fully discrete schemes are treated. The theory from the elliptic case as to when such estimates are asymptotically exact, on an element, is carried over to the error on an element at a given time. The basic principle is that the time-step error needs to be smaller than the space-discretization error. Numerical illustrations are given.
\end{abstract}

\section{INTRODUCTION}

Averaging techniques are widely used in order to gauge errors. In practice, they are attractive because they do not depend on the particular problem at hand, as residual methods do, and they are thus easy to implement as a post-processing to existing codes. When they work, they give error estimators, not merely error indicators; indeed, sometimes they are asymptotically exact. In the last few years research has been done to explain the success of averaging methods analytically for meshes which are not uniform. Most of this analytical work is done for elliptic problems, cf. Bank and Xu [1], [2], Carey [4], Hoffmann, Schatz, Wahlbin, and Wittum [7], and references therein.

The method of lines is a popular way to solve initial boundary value problems. Using this method the problem is first discretized in space by some method and the resulting system of ODEs is then approximated in time. A natural question to ask is whether the averaging technique error estimators work as well for time dependent problems.

This paper gives a positive answer in the case of a parabolic initial boundary value problem, provided the time discretization error is sufficiently small compared to the space discretization error. In fact, this caveat is natural: The basic averaging methods were developed for elliptic problems, and for the semidiscrete (timecontinuous and fictitious) problems it is well-known that the elliptic projection of the solution is superclose to the semidiscrete solution with respect to gradients. Thus, it seems plausible that the elliptic theory will essentially apply provided the time-discretization error is suitably controlled. These general observations were made by Ziukas and Wiberg in [14] and nicely illustrated numerically by them. For further references on analysis of parabolic a posteriori error estimates we refer the reader to the introduction in Lakkis and Makridakis [8].

To reiterate, in contrast to the elliptic case, we now have two main sources of the error, one from the space discretization and one from the time discretization. A

2000 Mathematics Subject Classification. 65M60, 65M20, 65M15.

Key words and phrases. a posteriori, finite element, fully discrete, pointwise estimates, parabolic second order equation. 
main technical difficulty is to unravel these two errors in a sufficiently distinct and separate fashion as to be able to apply the fine-tuned elliptic results at the single element level also to the parabolic case.

The plan of the paper is as follows. In Section 2 we state our main result. Section 3 is occupied with preliminary technical results and, as far as novel mathematics is concerned, forms the major part of the paper. The proof of the main result in Section 4 then follows essentially as in the elliptic case. The final Section 5 gives numerical illustrations. Familiarity with the elliptic case would be helpful to the reader.

\section{Statement of the Result}

Let $\Omega$ be a bounded domain in $\mathbb{R}^{N}, N \geq 1$, with a sufficiently smooth boundary. Consider the second order parabolic initial boundary value problem

$$
\begin{aligned}
u_{t}(x, t)+A u(x, t) & =f(x, t), \quad x \in \Omega, t>0, \\
u(x, 0) & =v(x), \quad x \in \Omega, \\
\frac{\partial u(x, t)}{\partial n} & =0, \quad x \in \partial \Omega, t>0 .
\end{aligned}
$$

For simplicity we assume $A=-\Delta+1$, where $\Delta=\sum \partial^{2} / \partial x_{j}^{2}$ is the Laplacian and 1 is the identity operator.

For the spatial finite element approximation of this problem, let $\mathcal{T}_{h}=\left\{\tau_{h}\right\}$, $0<h<1$, be a sequence of triangulations of $\Omega, \bar{\Omega}=\bigcup_{\tau \in \mathcal{T}} \bar{\tau}$, with the elements $\tau$ mutually disjoint. Furthermore, we assume that each $\tau$ that does not meet $\partial \Omega$ is an $N$-dimensional straight simplex, while those $\tau$ that meet $\partial \Omega$ are allowed to have lower-dimensional curved faces in common with $\partial \Omega$. The partitions are faceto-face so that simplices meet only in full lower-dimensional faces or not at all. The triangulations are assumed to be globally quasi-uniform, i.e. (if necessary after a renormalization of $h$ ),

$$
\operatorname{diam} \tau \leq h \leq C \text { (meas } \tau)^{1 / N}, \quad \forall \tau \in \mathcal{T}_{h} .
$$

Our finite element spaces are then the $\mathcal{C}^{0}$ simplicial Lagrange elements

$$
S_{h}=S_{h}^{r-1}(\Omega)=\left\{\chi \in \mathcal{C}(\bar{\Omega}):\left.\chi\right|_{\tau} \in \Pi^{r-1}(\tau)\right\},
$$

where $\Pi^{r-1}(D)$ denotes the set of polynomials of total degree $\leq r-1$ on $D$. Thus the usual inverse and approximation properties hold, which is really all we shall use. Of course, all the results from the literature that we use are valid under those assumptions.

We consider a single step fully discrete solution $U^{n}$ which is defined by

$$
U^{n+1}=r\left(k A_{h}\right) U^{n}+k\left(Q_{k h} P_{h} f\right)\left(t_{n}\right), \quad U^{0}=R_{h} v,
$$

where

$$
Q_{k h} P_{h} f(t)=\sum_{i=1}^{m} p_{i}\left(k A_{h}\right) P_{h} f\left(t+\tau_{i} k\right) .
$$

In the formulas above we have used the following notation:

- $k$ is a uniform time step, and $t_{n}=n k$ for any nonnegative integer $n$,

- $r(\lambda)$ and $p_{i}(\lambda)$ are rational functions defined on the spectrum of $k A_{h}$, and we further assume $r$ is $A$-stable and $r(\infty)=0$,

- $A_{h}: S_{h} \rightarrow S_{h}$ is defined by

$$
\left(A_{h} v, \chi\right)=(\nabla v, \nabla \chi)+(v, \chi), \text { for } \chi \in S_{h},
$$


- $P_{h}: L_{2}(\Omega) \rightarrow S_{h}$ is the $L_{2}$ projection defined by

$$
\left(P_{h} v, \chi\right)=(v, \chi) \text {, for } \chi \in S_{h},
$$

- elliptic projection $R_{h}: H^{1}(\Omega) \rightarrow S_{h}$ is given by

$$
\left(\nabla R_{h} v, \nabla \chi\right)+\left(R_{h} v, \chi\right)=(\nabla v, \nabla \chi)+(v, \chi), \text { for } \chi \in S_{h},
$$

- $\tau_{i}$ are distinct real numbers from the interval $[0,1]$.

To avoid the effect of unnatural boundary conditions and possible order reduction (cf. Brenner, Crouzeix, and Thomée [3]), we assume that the method (2.2) is both accurate and strictly accurate of order $q$, i.e. $r(\lambda)$ and $p_{i}(\lambda)$ satisfy the following properties as $\lambda \rightarrow 0$ :

$$
\begin{gathered}
r(\lambda)=e^{-\lambda}+O\left(\lambda^{q+1}\right), \\
\sum_{i=1}^{m} \tau_{i}^{l} p_{i}(\lambda)=\frac{l !}{(-\lambda)^{l+1}}\left(e^{-\lambda}-\sum_{j=0}^{l} \frac{(-\lambda)^{j}}{j !}\right)+O\left(\lambda^{q-l}\right), \quad \text { for } 0 \leq l \leq q,
\end{gathered}
$$

and

$$
\sum_{i=1}^{m} \tau_{i}^{l} p_{i}(\lambda)=\frac{l !}{(-\lambda)^{l+1}}\left(r(\lambda)-\sum_{j=0}^{l} \frac{(-\lambda)^{j}}{j !}\right), \text { for } 0 \leq l \leq q-1 .
$$

For the construction of such schemes, see [3] or Thomée [12], page 129. In fact, looking at these constructions, it is natural to assume also that the rational functions $p_{i}$ vanish at infinity,

$$
p_{i}(\lambda)=O\left(\frac{1}{\lambda}\right), \quad \text { as } \quad \lambda \rightarrow \infty .
$$

Let $d_{H}$ be a domain in $\Omega$ with a diameter comparable to $H$, where $H \geq h$. We assume that our recovered gradient operators $G_{H}: \mathcal{C}\left(d_{H}\right) \rightarrow \mathcal{C}\left(d_{H}\right)^{N}$ satisfy the following two conditions. First is a smoothing type estimate,

$$
\left\|G_{H} v\right\|_{L_{\infty}\left(d_{H}\right)} \leq C_{G} H^{-1}\|v\|_{L_{\infty}\left(d_{H}\right)}, \forall v \in W_{\infty}^{1}\left(\bar{d}_{H}\right),
$$

and then is the approximation property,

$$
\left\|\nabla v-G_{H} v\right\|_{L_{\infty}\left(d_{H}\right)} \leq C_{G} H^{r}\|v\|_{W_{\infty}^{r+1}\left(d_{H}\right)}, \forall v \in \mathcal{C}_{\infty}^{r+1}\left(\bar{d}_{H}\right) .
$$

Several examples of such operators are given in [7].

Our local error estimator is constructed as follows. Take any $\tau \in \mathcal{T}_{h}, \tau \subset d_{H}$ and set

$$
\mathcal{E}(\tau)=\left\|\nabla U^{n}-G_{H} U^{n}\right\|_{L_{\infty}(\tau)} .
$$

This is to be an estimator for $\left\|\nabla e^{n}\right\|_{L_{\infty}(\tau)}, e^{n}=U^{n}-u\left(t_{n}\right)$, the real gradient error on the element. The parameter $0<\varepsilon<1$, in the statement of the theorem below, is for you to choose, essentially to determine how close to pure approximation theory you want to be. With $\varepsilon$ near 1 , you are closest. Note that Alternative 1 ("nondegeneracy") or Alternative 2 ("degeneracy") below are tied to your choice of $\varepsilon$. Here is now our main result: 
Theorem 2.1. Let $r \geq 3$ and $0<\varepsilon<1$. Let $U^{n}$ be the one step fully discrete approximate solution to (2.1) at time $t_{n}=k n$ given by (2.2), which is both accurate and strictly accurate of order $q$, and $r(z)$ is A-stable with $r(\infty)=0$ and the $p_{i}$ satisfy (2.6). Then, provided $k^{q} \ll h^{r+1-\varepsilon}$, and $t_{n}$ is bounded, there exist constants $C_{1}$ and $C_{2}$ independent of $h$ and $k$ such that for each $\tau \in \mathcal{T}_{h}$, and with

$$
m=C_{1}\left(h / H+(H / h)^{r} h^{\varepsilon}\right),
$$

we have one of the following two alternatives:

Alternative 1. Suppose on the element $\tau$, the function $u$ satisfies the nondegeneracy condition

$$
\left|u\left(t_{n}\right)\right|_{W_{\infty}^{r}(\tau)} \geq h^{1-\varepsilon}\left(\left\|u\left(t_{n}\right)\right\|_{W_{\infty}^{r+1}(\Omega)}+\sup _{s \leq t_{n}}\left\|u_{t}(s)\right\|_{W_{\infty}^{r}(\Omega)}\right):=h^{1-\varepsilon}\left[u ; t_{n}\right] .
$$

In this case

$$
\begin{gathered}
\left\|\nabla u\left(t_{n}\right)-G_{H} U^{n}\right\|_{L_{\infty}(\tau)} \leq m\left\|\nabla e^{n}\right\|_{L_{\infty}(\tau)}, \\
\mathcal{E}(\tau) \leq(1+m)\left\|\nabla e^{n}\right\|_{L_{\infty}(\tau)},
\end{gathered}
$$

and, if $m<1$,

$$
\frac{1}{1+m} \mathcal{E}(\tau) \leq\left\|\nabla e^{n}\right\|_{L_{\infty}(\tau)} \leq \frac{1}{1-m} \mathcal{E}(\tau) .
$$

If $H=H(h)$ is chosen so that $m \rightarrow 0$ as $h \rightarrow 0$, the estimator is asymptotically exact.

Alternative 2. Suppose (2.9) does not hold, i.e.

$$
\left|u\left(t_{n}\right)\right|_{W_{\infty}^{r}(\tau)}<h^{1-\varepsilon}\left[u ; t_{n}\right] .
$$

In this case

$$
\begin{gathered}
\left\|\nabla e^{n}\right\|_{L_{\infty}(\tau)} \leq C_{2} h^{r-\varepsilon}\left[u ; t_{n}\right], \\
\left\|\nabla u\left(t_{n}\right)-G_{H} U^{n}\left(t_{n}\right)\right\|_{L_{\infty}(\tau)} \leq m h^{r-\varepsilon}\left[u ; t_{n}\right],
\end{gathered}
$$

and

$$
\mathcal{E}(\tau) \leq\left(m+C_{2}\right) h^{r-\varepsilon}\left[u ; t_{n}\right]
$$

Thus in the "degenerate" Alternative 2, both the real error and the error estimator are very small, but there may be no connection between them. (cf. our numerical illustration in Section 5, Example 4).

Remark 1. To keep this paper short, we only consider approximation degree $r \geq$ 3 and recovered gradient operators $G_{H}$ that satisfy (2.7) and (2.8). As for the piecewise linear case, $r=2$, it could have been included using the elliptic results of [11]. The quadrature weighted Zienkiewicz-Zhu (ZZ) operator, which fails to satisfy the smoothing property (2.7), could also been included using the elliptic results of [4]. However, the most widely used form of the $\mathrm{ZZ}$ operator, the unweighted one, still eludes serious analysis except for meshes with certain degrees of uniformity where superconvergence phenomena can be used in the analysis. 


\section{Preliminary Results}

In this section we collect results which are essential in order to prove the main result of the paper. We will use the notation

$$
\ell_{h}=|\log h|
$$

throughout the paper.

The first result is pointwise estimates for the Green's function for the continuous problem (2.1).

Lemma 3.1. If $f \equiv 0$, the solution of (2.1) may be represented in terms of a Green's function $G(x, y ; t, 0), t>0, x, y \in \Omega$, as

$$
u(x, t)=\int_{\Omega} G(x, y ; t, 0) v(y) d y .
$$

Assume that the boundary $\partial \Omega$ is sufficiently smooth. Then for any integer $l_{0}$ and multi-integer $l$, there exist constants $C$ and $c>0$ such that for the Green's function $G(x, y ; t, s), 0 \leq s<t$, and $x, y \in \Omega$, we have

$$
\left|D_{t}^{l_{0}} D_{x}^{l} G(x, y ; t, s)\right| \leq C(|x-y|+\sqrt{t-s})^{-\left(N+2 l_{0}+|l|\right)} e^{-c \frac{|x-y|^{2}}{t-s}} .
$$

A proof is given in Ė̀̆del'man and Ivasišen [5].

The next result that we need is Lemma 3.3 in [7].

Lemma 3.2. There exists a constant $\widehat{C}_{1}$ independent of $h$ such that

$$
h^{r-1}\left|u\left(t_{n}\right)\right|_{W_{\infty}^{r}(\tau)}-h^{r}\left\|u\left(t_{n}\right)\right\|_{W_{\infty}^{r+1}(\tau)} \leq \widehat{C}_{1}\left\|\nabla e^{n}\right\|_{L_{\infty}(\tau)} .
$$

In the next lemma we will show stability and smoothing type estimates of the homogeneous solution operator $E_{h}(t)$ for the semicontinuous problem in the $W_{\infty}^{1}$ norm.

Lemma 3.3. Let $E_{h}(t)=e^{-A_{h} t}$. There exists a constant $C$ independent of $h$ such that

$$
\left\|E_{h}(t) v\right\|_{W_{\infty}^{1}(\Omega)}+t \ell_{h}^{-1}\left\|E_{h}^{\prime}(t) v\right\|_{W_{\infty}^{1}(\Omega)} \leq C\|v\|_{W_{\infty}^{1}(\Omega)}, \quad \forall v \in S_{h}, t \geq 0 .
$$

Proof. Let $u(t)=e^{-A t} v$ and $u_{h}(t)=e^{-A_{h} t} v$, where $v \in S_{h}$. By the triangle inequality,

$$
\left\|u_{h}(t)\right\|_{W_{\infty}^{1}(\Omega)} \leq\left\|u_{h}(t)-u(t)\right\|_{W_{\infty}^{1}(\Omega)}+\|u(t)\|_{W_{\infty}^{1}(\Omega)} .
$$

With a suitable $\chi \in S_{h}$, using the triangle and inverse inequalities, we have

$$
\begin{aligned}
\left\|u_{h}(t)-u(t)\right\|_{W_{\infty}^{1}(\Omega)} & \leq\left\|u_{h}(t)-\chi\right\|_{W_{\infty}^{1}(\Omega)}+\|\chi-u(t)\|_{W_{\infty}^{1}(\Omega)} \\
& \leq \frac{C}{h}\left\|u_{h}(t)-\chi\right\|_{L_{\infty}(\Omega)}+\|\chi-u(t)\|_{W_{\infty}^{1}(\Omega)} \\
& \leq \frac{C}{h}\left\|u_{h}(t)-u(t)\right\|_{L_{\infty}(\Omega)}+\frac{C}{h}\|u(t)-\chi\|_{L_{\infty}(\Omega)}+\|\chi-u(t)\|_{W_{\infty}^{1}(\Omega)} \\
& \leq \frac{C}{h}\left\|u_{h}(t)-u(t)\right\|_{L_{\infty}(\Omega)}+C\|u(t)\|_{W_{\infty}^{1}(\Omega)} .
\end{aligned}
$$

Thus,

$$
\left\|u_{h}(t)\right\|_{W_{\infty}^{1}(\Omega)} \leq \frac{C}{h}\left\|u_{h}(t)-u(t)\right\|_{L_{\infty}(\Omega)}+C\|u(t)\|_{W_{\infty}^{1}(\Omega)} .
$$


By Corollary 2.4 in [10], $\left\|u_{h}(t)-u(t)\right\|_{L_{\infty}(\Omega)} \leq C h\|u\|_{W_{\infty}^{1}\left(Q_{t}\right)}$, where $Q_{t}=\Omega \times[0, t]$. Hence,

$$
\left\|u_{h}(t)\right\|_{W_{\infty}^{1}(\Omega)} \leq C\|u\|_{W_{\infty}^{1}\left(Q_{t}\right)} .
$$

Next we will show $\|u\|_{W_{\infty}^{1}\left(Q_{t}\right)} \leq C\|v\|_{W_{\infty}^{1}(\Omega)}$.

Given any initial value $v$ the corresponding solution $u$ to the homogeneous problem can be represented using the Green's function as

$$
u(x, t)=\int_{\Omega} G(x, y ; t, 0) v(y) d y,
$$

and hence

$$
D_{x} u(x, t)=\int_{\Omega} D_{x} G(x, y ; t, 0) v(y) d y .
$$

It is easy to see that if $v \equiv 1$, then $u(x, t)=e^{-t}$, and

$$
\int_{\Omega} D_{x} G(x, y ; t, 0) d y=0 .
$$

Thus

$$
D_{x} u(x, t)=\int_{\Omega} D_{x} G(x, y ; t, 0) v(y) d y=\int_{\Omega} D_{x} G(x, y ; t, 0)(v(y)-v(x)) d y .
$$

By the Mean Value Theorem and the Green's function estimate in Lemma 3.1, we have

$$
\left|D_{x} u(x, t)\right| \leq C\|v\|_{W_{\infty}^{1}(\Omega)} \int_{\Omega} \frac{e^{-c \frac{|x-y|^{2}}{t}}|y-x|}{(|x-y|+\sqrt{t})^{N+1}} d y \leq C\|v\|_{W_{\infty}^{1}(\Omega)},
$$

which proves the stability of $E_{h}(t)$.

Next we will show the smoothing property.

Let $w_{h}(t)=t u_{h, t}(t)$ and $w(t)=t u_{t}(t)$. Then

$$
w_{h, t}+A_{h} w_{h}=u_{h, t}, \quad w_{h}(0)=0,
$$

and

$$
w_{t}+A w=u_{t}, \quad w(0)=0 .
$$

Let $\eta:=w_{h}-R_{h} w$. Since $\left.\frac{\partial w}{\partial n}\right|_{\partial \Omega}=0$, we have $P_{h} A w=A_{h} R_{h} w$. Thus $\eta \in S_{h}$ satisfies

$$
\begin{aligned}
& \eta_{t}+A_{h} \eta=\left(P_{h}-R_{h}\right) w_{t}+\left(u_{h}-P_{h} u\right)_{t} \\
& \eta(0)=0 .
\end{aligned}
$$

By Duhamel's principle

$$
\eta(t)=\int_{0}^{t} E_{h}(t-s)\left[\left(P_{h}-R_{h}\right) w+\left(u_{h}-P_{h} u\right)\right]_{t}(s) d s .
$$

Integrating by parts and using that $v \in S_{h}$ and $w(0)=0$, we have

$$
\begin{aligned}
\eta(t) & =\int_{0}^{t} E_{h}^{\prime}(t-s)\left[\left(P_{h}-R_{h}\right) w+\left(u_{h}-P_{h} u\right)\right](s) d s+\left(P_{h}-R_{h}\right) w(t)+\left(u_{h}-P_{h} u\right)(t) \\
& =I_{1}+I_{2}+I_{3} .
\end{aligned}
$$


By Theorem 2.1 in [10], $\left\|E_{h}^{\prime}(t)\right\|_{L_{\infty}} \leq \frac{C}{t+h^{2}}$. Also using Corollary 2.4 in [10] for $\left(u_{h}-P_{h} u\right)(t)$ and the already proved stability result, we have

$$
\begin{aligned}
\left\|I_{1}\right\|_{L_{\infty}(\Omega)} & \leq \int_{0}^{t} \frac{C}{t-s+h^{2}}\left[h\|w\|_{W_{\infty}^{1}\left(Q_{t}\right)}+h\|u\|_{W_{\infty}^{1}\left(Q_{t}\right)}\right] d s \\
& \leq C \ell_{h} h\left(\|w\|_{W_{\infty}^{1}\left(Q_{t}\right)}+\|u\|_{W_{\infty}^{1}\left(Q_{t}\right)}\right) \leq C \ell_{h} h\left(\|w\|_{W_{\infty}^{1}\left(Q_{t}\right)}+\|v\|_{W_{\infty}^{1}(\Omega)}\right) .
\end{aligned}
$$

Similarly

$$
\left\|I_{2}\right\|_{L_{\infty}(\Omega)}+\left\|I_{3}\right\|_{L_{\infty}(\Omega)} \leq C \ell_{h} h\left(\|w\|_{W_{\infty}^{1}\left(Q_{t}\right)}+\|v\|_{W_{\infty}^{1}(\Omega)}\right) .
$$

Thus, by an inverse estimate,

$$
\|\eta(t)\|_{W_{\infty}^{1}(\Omega)} \leq C h^{-1}\|\eta(t)\|_{L_{\infty}(\Omega)} \leq C \ell_{h}\left(\|w\|_{W_{\infty}^{1}\left(Q_{t}\right)}+\|v\|_{W_{\infty}^{1}(\Omega)}\right) .
$$

Since $w_{h}=\eta+R_{h} w$ and we have stability of $R_{h}$ in the $W_{\infty}^{1}$ norm, the only thing that is left is to estimate $\|w(t)\|_{W_{\infty}^{1}(\Omega)}$. From (3.1),

$$
\begin{aligned}
D_{t} D_{x} u(x, t) & =\int_{\Omega} D_{t} D_{x} G(x, y ; t, 0)(v(y)-v(x)) d y \\
& \leq C\|v\|_{W_{\infty}^{1}(\Omega)} \int_{\Omega} \frac{e^{-c \frac{|x-y|^{2}}{t}}|y-x|}{(|x-y|+\sqrt{t})^{N+3}} d y \leq \frac{C}{t}\|v\|_{W_{\infty}^{1}(\Omega)} .
\end{aligned}
$$

Hence, $\|w\|_{W_{\infty}^{1}\left(Q_{t}\right)} \leq C\|v\|_{W_{\infty}^{1}(\Omega)}$ and we have the smoothing property

$$
\left\|E_{h}^{\prime}(t) v\right\|_{W_{\infty}^{1}(\Omega)} \leq C \frac{\ell_{h}}{t}\|v\|_{W_{\infty}^{1}(\Omega)} .
$$

This completes the proof of Lemma 3.3.

Remark 2. Actually, in our present work, we only use the stability part of Lemma 3.3. We have included the smoothing part for completeness and possible future references.

Our next lemma is an intermediate derivatives estimate on the finite element spaces (1, between 2 and 0 ). The second derivatives on the finite element spaces are interpreted in the sense of $A_{h}$; a logarithmic factor appears due to this.

Lemma 3.4. There exists a constant $C$ independent of $h$, such that for all $0<\delta \leq$ 1 and $\chi \in S_{h}$,

$$
\|\chi\|_{W_{\infty}^{1}(\Omega)} \leq C \ell_{h}\left((h+\delta)\left\|A_{h} \chi\right\|_{L_{\infty}(\Omega)}+\frac{1}{\delta+h}\|\chi\|_{L_{\infty}(\Omega)}\right) .
$$

Proof. Using an inverse estimate, for any $2<p<\infty$,

$$
\|\chi\|_{W_{\infty}^{1}(\Omega)} \leq C h^{-N / p}\|\chi\|_{W_{p}^{1}(\Omega)} \text {, for any } \chi \in S_{h} .
$$

Now with $T=A^{-1}$ and $T_{h}=A_{h}^{-1} P_{h}$, using approximation properties of the elliptic projection and elliptic regularity theory,

$$
\begin{aligned}
\|\chi\|_{W_{p}^{1}(\Omega)} & =\left\|T_{h} A_{h} \chi\right\|_{W_{p}^{1}(\Omega)} \leq\left\|\left(T_{h}-T\right) A_{h} \chi\right\|_{W_{p}^{1}(\Omega)}+\left\|T A_{h} \chi\right\|_{W_{p}^{1}(\Omega)} \\
& \leq C h\left\|T A_{h} \chi\right\|_{W_{p}^{2}(\Omega)}+\left\|T A_{h} \chi\right\|_{W_{p}^{1}(\Omega)} \leq C h p\left\|A_{h} \chi\right\|_{L_{\infty}(\Omega)}+\left\|T A_{h} \chi\right\|_{W_{p}^{1}(\Omega)} .
\end{aligned}
$$

From (3.2), then

$$
\|\chi\|_{W_{\infty}^{1}(\Omega)} \leq C h^{-N / p}\left(C h p\left\|A_{h} \chi\right\|_{L_{\infty}(\Omega)}+\left\|T A_{h} \chi\right\|_{W_{p}^{1}(\Omega)}\right) .
$$


For any $0<\delta \leq 1$, by standard intermediate derivatives estimates and again elliptic regularity,

$$
\begin{aligned}
\left\|T A_{h} \chi\right\|_{W_{p}^{1}(\Omega)} & \leq C\left(\delta\left\|T A_{h} \chi\right\|_{W_{p}^{2}(\Omega)}+\frac{1}{\delta}\left\|T A_{h} \chi\right\|_{L_{p}(\Omega)}\right) \\
& \leq C\left(\delta p\left\|A_{h} \chi\right\|_{L_{\infty}(\Omega)}+\frac{1}{\delta}\left\|T A_{h} \chi\right\|_{L_{p}(\Omega)}\right),
\end{aligned}
$$

so that using (3.3),

$$
\|\chi\|_{W_{\infty}^{1}(\Omega)} \leq C h^{-N / p}\left((\delta+h) p\left\|A_{h} \chi\right\|_{L_{\infty}(\Omega)}+\frac{1}{\delta}\left\|T A_{h} \chi\right\|_{L_{p}(\Omega)}\right) .
$$

Finally,

$$
\begin{aligned}
\left\|T A_{h} \chi\right\|_{L_{p}(\Omega)} & \leq\left\|\left(T-T_{h}\right) A_{h} \chi\right\|_{L_{p}(\Omega)}+\|\chi\|_{L_{p}(\Omega)} \\
& \leq C h^{2} p\left\|A_{h} \chi\right\|_{L_{\infty}(\Omega)}+\|\chi\|_{L_{\infty}(\Omega)} \leq C p\|\chi\|_{L_{\infty}(\Omega)} .
\end{aligned}
$$

Thus, from (3.4), choosing $p=\ell_{h}$,

$$
\|\chi\|_{W_{\infty}^{1}(\Omega)} \leq C \ell_{h}\left((\delta+h)\left\|A_{h} \chi\right\|_{L_{\infty}(\Omega)}+\frac{1}{\delta}\|\chi\|_{L_{\infty}(\Omega)}\right) .
$$

Of course, if $\delta \leq h$, then by an inverse estimate we have

$$
\|\chi\|_{W_{\infty}^{1}(\Omega)} \leq \frac{C}{h}\|\chi\|_{L_{\infty}(\Omega)} .
$$

This completes the proof of the lemma.

The next result shows a localized property of the gradient of the error of the fully discrete solution.

Proposition 3.5. Let $u$ satisfy (2.1) and let $U^{n}$ be the fully discrete solution computed at time $t_{n}=n k$ by (2.2). Then, for any $0<\varepsilon<1$, there exists a constant $C_{\varepsilon}$ independent of $u, k$ and $h$, such that for any $x \in \Omega$,

$$
\begin{aligned}
\left|\nabla e^{n}(x)\right| & \leq C_{\varepsilon} h^{r-1}\left(\sum_{|\alpha|=r}\left|D_{x}^{\alpha} u\left(x, t_{n}\right)\right|+h^{1-\varepsilon}\left\|u\left(t_{n}\right)\right\|_{W_{\infty}^{r+1}(\Omega)}\right) \\
& +C \ell_{h} h^{r} \sqrt{t_{n}} \sup _{s \leq t_{n}}\left\|u_{t}(s)\right\|_{W_{\infty}^{r}(\Omega)}+\boldsymbol{P}_{1},
\end{aligned}
$$

where

$$
\boldsymbol{P}_{1}=C k^{q} \int_{0}^{t_{n}}\left(\left\|A u^{(q)}(s)\right\|_{W_{\infty}^{1}(\Omega)}+\left\|u^{(q+1)}(s)\right\|_{W_{\infty}^{1}(\Omega)}\right) d s .
$$

Proof. Using the triangle inequality we have

$\left|\nabla\left(u\left(x, t_{n}\right)-U^{n}(x)\right)\right| \leq\left|\nabla\left(u\left(x, t_{n}\right)-R_{h} u\left(x, t_{n}\right)\right)\right|+\left|\nabla\left(R_{h} u\left(x, t_{n}\right)-U^{n}(x)\right)\right|$.

From Theorem 4.2 in [9], for any $0<\varepsilon<1$,

$$
\left|\nabla\left(u\left(x, t_{n}\right)-R_{h} u\left(x, t_{n}\right)\right)\right| \leq C_{\varepsilon} h^{r-1}\left(\sum_{|\alpha|=r}\left|D_{x}^{\alpha} u\left(x, t_{n}\right)\right|+h^{1-\varepsilon}\left\|u\left(t_{n}\right)\right\|_{W_{\infty}^{r+1}(\Omega)}\right) .
$$

In order to show the desired bound for $\left|\nabla\left(R_{h} u\left(x, t_{n}\right)-U^{n}(x)\right)\right|$, we will follow the proof of Theorem 9.6 in [12]. First we note that $w_{h}=R_{h} u$ satisfies

$$
w_{h, t}+A_{h} w_{h}=R_{h} u_{t}+P_{h} A u=P_{h}\left(f+\rho_{t}\right):=g_{h},
$$


where $\rho=R_{h} u-u$.

Let

$$
W^{n+1}=r\left(k A_{h}\right) W^{n}+k\left(Q_{k h} g_{h}\right)\left(t_{n}\right), W^{0}=R_{h} v,
$$

be the corresponding fully discrete solution to $w_{h}$. Since by Lemma $3.3, E_{h}(t)$ is stable in $W_{\infty}^{1}$ norm, from Theorem 1 in [3] with $p_{0}=q$, we have

$$
\left\|W^{n}-w_{h}\left(t_{n}\right)\right\|_{W_{\infty}^{1}(\Omega)} \leq C k^{q} \int_{0}^{t_{n}}\left(\left\|A_{h} w_{h}^{(q)}(s)\right\|_{W_{\infty}^{1}(\Omega)}+\left\|w_{h}^{(q+1)}(s)\right\|_{W_{\infty}^{1}(\Omega)}\right) d s .
$$

Since $A_{h} R_{h}=P_{h} A$ and the fact that $R_{h}$ and $P_{h}$ are stable in $W_{\infty}^{1}$ norm, we have

$$
\left\|W^{n}-w_{h}\left(t_{n}\right)\right\|_{W_{\infty}^{1}(\Omega)} \leq C k^{q} \int_{0}^{t_{n}}\left(\left\|A u^{(q)}(s)\right\|_{W_{\infty}^{1}(\Omega)}+\left\|u^{(q+1)}(s)\right\|_{W_{\infty}^{1}(\Omega)}\right) d s .
$$

It remains to consider $Z^{n}=U^{n}-W^{n}$, which satisfies

$$
Z^{n+1}=r\left(k A_{h}\right) Z^{n}+k\left(Q_{k h} P_{h} \rho_{t}\right)\left(t_{n}\right), \quad Z^{0}=0 .
$$

Thus,

$$
\left\|Z^{n}\right\|_{W_{\infty}^{1}(\Omega)} \leq k\left\|Q_{k h} P_{h} \rho_{t}\left(t_{n-1}\right)\right\|_{W_{\infty}^{1}(\Omega)}+k \sum_{j=0}^{n-2}\left\|r\left(k A_{h}\right)^{n-1-j} Q_{k h} P_{h} \rho_{t}\left(t_{j}\right)\right\|_{W_{\infty}^{1}(\Omega)} .
$$

We start by bounding the sum on the right. First we will show the following smoothing type estimate

$$
\left\|r^{n}\left(k A_{h}\right) \chi\right\|_{W_{\infty}^{1}(\Omega)} \leq C \frac{\ell_{h}}{\sqrt{k n}}\|\chi\|_{L_{\infty}(\Omega)}, \text { for any } \chi \in S_{h} .
$$

We note that, by the result of [10], $A_{h}$ satisfies a resolvent estimate in $L_{\infty}$ norm which is logarithm free. Hence from Theorem 2.2 in Hansbo [6],

$$
\left\|A_{h} r^{n}\left(k A_{h}\right) \chi\right\|_{L_{\infty}(\Omega)} \leq \frac{C}{k n}\|\chi\|_{L_{\infty}(\Omega)},
$$

and since $r(\lambda)$ is A-stable, by Theorem 8.2 in [12],

$$
\left\|r^{n}\left(k A_{h}\right) \chi\right\|_{L_{\infty}(\Omega)} \leq C\|\chi\|_{L_{\infty}(\Omega)} .
$$

Taking $\delta=\sqrt{n k+h^{2}}$ in Lemma 3.4 proves (3.6).

Using (3.6), the boundedness of $Q_{k h}$ and of $P_{h}$ in $L_{\infty}$ norm, we obtain

$$
\begin{aligned}
& k \sum_{j=0}^{n-2}\left\|r\left(k A_{h}\right)^{n-1-j} Q_{k h} P_{h} \rho_{t}\left(t_{j}\right)\right\|_{W_{\infty}^{1}(\Omega)} \leq C \ell_{h} k \sum_{j=0}^{n-2} \frac{\sup _{t_{j} \leq s \leq t_{j+1}}\left\|\rho_{t}(s)\right\|_{L_{\infty}(\Omega)}}{\sqrt{k(n-1-j)}} \\
& \leq C \ell_{h} h^{r} \sqrt{k} \sup _{s \leq t_{n}}\left\|u_{t}(s)\right\|_{W_{\infty}^{r}(\Omega)} \sum_{j=0}^{n-2} \frac{1}{\sqrt{n-1-j}} \leq C \ell_{h} h^{r} \sqrt{t_{n}} \sup _{s \leq t_{n}}\left\|u_{t}(s)\right\|_{W_{\infty}^{r}(\Omega)} .
\end{aligned}
$$

To bound the first term on the right in (3.5), we recall (2.6), which implies similarly to (3.6) that

$$
\begin{aligned}
k\left\|Q_{k h} P_{h} \rho_{t}\left(t_{n-1}\right)\right\|_{W_{\infty}^{1}(\Omega)} & \leq C \ell_{h} \sqrt{k} \sup _{t_{n-1} \leq s \leq t_{n}}\left\|P_{h} \rho_{t}(s)\right\|_{L_{\infty}(\Omega)} \\
& \leq C \ell_{h} \sqrt{k} h^{r} \sup _{t_{n-1} \leq s \leq t_{n}}\left\|u_{t}(s)\right\|_{W_{\infty}^{r}(\Omega)} .
\end{aligned}
$$

Thus we have shown the desired bound for $\left\|Z^{n}\right\|_{W_{\infty}^{1}(\Omega)}$, and the proof of the proposition is complete. 
We shall also need the similar result for function values.

Proposition 3.6. There exists a constant $C_{\varepsilon}$ independent of $u, k$ and $h$, such that for any $x \in \Omega$,

$$
\begin{aligned}
\left|e^{n}(x)\right| & \leq C h^{r}\left(\sum_{|\alpha|=r}\left|D_{x}^{\alpha} u\left(x, t_{n}\right)\right|+h^{1-\varepsilon}\left\|u\left(t_{n}\right)\right\|_{W_{\infty}^{r+1}(\Omega)}\right) \\
& +C h^{r+1} \log (n+1) \sup _{s \leq t_{n}}\left\|u_{t}(s)\right\|_{W_{\infty}^{r}(\Omega)}+\boldsymbol{P}_{2},
\end{aligned}
$$

where

$$
\boldsymbol{P}_{2}=C k^{q} \int_{0}^{t_{n}}\left(\left\|A u^{(q)}(s)\right\|_{L_{\infty}(\Omega)}+\left\|u^{(q+1)}(s)\right\|_{L_{\infty}(\Omega)}\right) d s .
$$

Proof. Similarly to the proof of the previous proposition, from the triangle inequality we have

$$
\left|u\left(x, t_{n}\right)-U^{n}(x)\right| \leq\left|u\left(x, t_{n}\right)-R_{h} u\left(x, t_{n}\right)\right|+\left|R_{h} u\left(x, t_{n}\right)-U^{n}(x)\right| .
$$

By Theorem 4.1 in [9], for any $0<\varepsilon<1$,

$$
\left|u\left(x, t_{n}\right)-R_{h} u\left(x, t_{n}\right)\right| \leq C h^{r}\left(\sum_{|\alpha|=r}\left|D_{x}^{\alpha} u\left(x, t_{n}\right)\right|+h^{1-\varepsilon}\left\|u\left(t_{n}\right)\right\|_{W_{\infty}^{r+1}(\Omega)}\right) .
$$

To bound $\left|R_{h} u\left(x, t_{n}\right)-U^{n}(x)\right|$, we again proceed analogously to the proof of the previous proposition. Thus we obtain

$$
\left\|W^{n}-w_{h}\left(t_{n}\right)\right\|_{L_{\infty}(\Omega)} \leq C k^{q} \int_{0}^{t_{n}}\left(\left\|A u^{(q)}(s)\right\|_{L_{\infty}(\Omega)}+\left\|u^{(q+1)}(s)\right\|_{L_{\infty}(\Omega)}\right) d s
$$

where $w_{h}=R_{h} u$ and $W^{n}$ is its fully discrete solution.

Now $Z^{n}=U^{n}-W^{n}$ with $\rho=R_{h} u-u$ satisfies

$$
Z^{n}=k \sum_{j=0}^{n-1} r\left(k A_{h}\right)^{n-j-1} Q_{k h} P_{h} \rho_{t}\left(t_{j}\right)
$$

Thus,

$$
\begin{aligned}
\left\|Z^{n}\right\|_{L_{\infty}(\Omega)} & \leq k\left\|Q_{k h} P_{h} \rho_{t}\left(t_{n-1}\right)\right\|_{L_{\infty}(\Omega)}+k \sum_{j=0}^{n-2}\left\|r\left(k A_{h}\right)^{n-1-j} Q_{k h} P_{h} \rho_{t}\left(t_{j}\right)\right\|_{L_{\infty}(\Omega)} \\
& =I_{1}+I_{2} .
\end{aligned}
$$

To bound the sum, we use that $Q_{k h}$ and $A_{h}$ commute and the operator identity $A_{h}^{-1} P_{h}=R_{h} A^{-1}$. Hence we can write

$$
I_{2}=k \sum_{j=0}^{n-2}\left\|A_{h} r\left(k A_{h}\right)^{n-1-j} Q_{k h} R_{h} A^{-1} \rho_{t}\left(t_{j}\right)\right\|_{L_{\infty}(\Omega)} .
$$

Recalling (3.7),

$$
\left\|A_{h} r^{n}\left(k A_{h}\right) \chi\right\|_{L_{\infty}(\Omega)} \leq \frac{C}{k n}\|\chi\|_{L_{\infty}(\Omega)},
$$


and using the boundedness of $Q_{k h}$ and $R_{h}$ in $L_{\infty}$ norm for $r \geq 3$, we have

$$
I_{2} \leq C \sum_{j=0}^{n-2} \frac{\sup _{t_{j} \leq s \leq t_{j+1}}\left\|A^{-1} \rho_{t}(s)\right\|_{L_{\infty}(\Omega)}}{n-1-j} .
$$

Next we will estimate $\left\|A^{-1} \rho_{t}(s)\right\|_{L_{\infty}(\Omega)}$. Setting up a duality argument, we write

$$
\left\|A^{-1}\left(R_{h}-I\right) v\right\|_{L_{\infty}}=\sup _{\|\psi\|_{L_{1}}=1}\left(A^{-1}\left(R_{h}-I\right) v, \psi\right) .
$$

Using that $A^{-1}$ is self adjoint and the definition of the elliptic projection, we have for any $\chi \in S_{h}$ and fixed $\psi$,

$$
\begin{aligned}
& \left(A^{-1}\left(R_{h}-I\right) v, \psi\right)=\left(\left(R_{h}-I\right) v, A^{-1} \psi\right)=\left(\left(R_{h}-I\right) v, A A^{-2} \psi\right) \\
& =\left(\nabla\left(R_{h}-I\right) v, \nabla\left(A^{-2} \psi-\chi\right)\right)+\left(\left(R_{h}-I\right) v, A^{-2} \psi-\chi\right) \\
& \leq\left\|R_{h} v-v\right\|_{W_{\infty}^{1}(\Omega)}\left\|A^{-2} \psi-\chi\right\|_{W_{1}^{1}(\Omega)} .
\end{aligned}
$$

By approximation theory, we find that

$$
\left\|A^{-1} \rho_{t}(s)\right\|_{L_{\infty}(\Omega)} \leq C h^{r-1}\left\|u_{t}(s)\right\|_{W_{\infty}^{r}(\Omega)} C h^{2}\left\|A^{-2} \psi\right\|_{W_{1}^{3}(\Omega)} \leq C h^{r+1}\left\|u_{t}(s)\right\|_{W_{\infty}^{r}(\Omega)} .
$$

Hence

$$
I_{2} \leq C h^{r+1} \log (n+1) \sup _{s \leq t_{n}}\left\|u_{t}(s)\right\|_{W_{\infty}^{r}(\Omega)} .
$$

For the first term on the right of (3.8), we proceed similarly using also (2.6):

$$
\begin{aligned}
I_{1} & \leq k\left\|Q_{k h} P_{h} \rho_{t}\left(t_{n-1}\right)\right\|_{L_{\infty}(\Omega)}=k\left\|A_{h} Q_{k h} R_{h} A^{-1} \rho_{t}\left(t_{n-1}\right)\right\|_{L_{\infty}(\Omega)} \\
& \leq C \sup _{t_{n-1} \leq s \leq t_{n}}\left\|A^{-1} \rho_{t}(s)\right\|_{L_{\infty}(\Omega)} \leq C h^{r+1} \sup _{t_{n-1} \leq s \leq t_{n}}\left\|u_{t}(s)\right\|_{W_{\infty}^{r}(\Omega)} .
\end{aligned}
$$

Hence from (3.8), (3.9), and (3.10),

$$
\left\|Z^{n}\right\|_{L_{\infty}(\Omega)} \leq C h^{r+1} \log (n+1) \sup _{s \leq t_{n}}\left\|u_{t}(s)\right\|_{W_{\infty}^{r}(\Omega)}
$$

and the proof of the proposition is complete.

\section{Proof of the Main Results}

Using our preparation from the previous section, we can now prove Theorem 2.1. The exact meaning of $k^{q}<<h^{r+1-\varepsilon}$ is the assumption that the terms $\mathbf{P}_{1}$ and $\mathbf{P}_{2}$ can be dropped from the estimates in Propositions 3.5 and 3.6.

Proof. Recall the notation

$$
\left[u ; t_{n}\right]:=\left\|u\left(t_{n}\right)\right\|_{W_{\infty}^{r+1}(\Omega)}+\sup _{s \leq t_{n}}\left\|u_{t}(s)\right\|_{W_{\infty}^{r}(\Omega)} .
$$

We assume that the final time $t_{n}$ is bounded, and the time discretization is sufficiently fine, i.e. $k^{q} \ll h^{r+1-\varepsilon}$. If we thus neglect in Propositions 3.5 and 3.6 all terms involving the time discretization step-size $k$, we have

$$
\left|\nabla e^{n}(x)\right| \leq \widehat{C} h^{r-1}\left(\sum_{|\alpha|=r}\left|D_{x}^{\alpha} u\left(x, t_{n}\right)\right|+h^{1-\varepsilon}\left[u ; t_{n}\right]\right) .
$$


and

$$
\left|e^{n}(x)\right| \leq \widehat{C} h^{r}\left(\sum_{|\alpha|=r}\left|D_{x}^{\alpha} u\left(x, t_{n}\right)\right|+h^{1-\varepsilon}\left[u ; t_{n}\right]\right) .
$$

Next we will show (2.10). By the triangle inequality, (2.7), and (2.8), we have

$$
\begin{aligned}
\left|\nabla u\left(t_{n}\right)(x)-G_{H} U^{n}(x)\right| & \leq\left|\nabla u\left(t_{n}\right)(x)-G_{H} u\left(t_{n}\right)(x)\right|+\left|G_{H} e^{n}(x)\right| \\
& \leq C_{G} H^{r}\left\|u\left(t_{n}\right)\right\|_{W_{\infty}^{r+1}(\Omega)}+C_{G} H^{-1}\left\|e^{n}\right\|_{L_{\infty}\left(d_{H}\right)} \\
& \leq C_{G} H^{r}\left[u ; t_{n}\right]+C_{G} H^{-1}\left\|e^{n}\right\|_{L_{\infty}\left(d_{H}\right)} .
\end{aligned}
$$

Let $\bar{x} \in d_{H}$ be the point such that $\left\|e^{n}\right\|_{L_{\infty}\left(d_{H}\right)}=\left|e^{n}(\bar{x})\right|$. From (4.2) and the Mean Value Theorem,

$$
\begin{aligned}
\left|e^{n}(\bar{x})\right| & \leq \widehat{C} h^{r}\left(\sum_{|\alpha|=r}\left|D_{x}^{\alpha} u\left(\bar{x}, t_{n}\right)\right|+h^{1-\varepsilon}\left[u ; t_{n}\right]\right) \\
& \leq \widehat{C} h^{r}\left(\left|u\left(t_{n}\right)\right|_{W_{\infty}^{r}(\tau)}+\left(H+h^{1-\varepsilon}\right)\left[u ; t_{n}\right]\right) .
\end{aligned}
$$

Combining the last two equations and using that $H \geq h$, we have

$$
\begin{aligned}
\left|\nabla u\left(t_{n}\right)(x)-G_{H} U^{n}(x)\right| & \leq C_{G} H^{r}\left[u ; t_{n}\right]+\widehat{C} C_{G} \frac{h^{r}}{H}\left(\left|u\left(t_{n}\right)\right|_{W_{\infty}^{r}(\tau)}+\left(H+h^{1-\varepsilon}\right)\left[u ; t_{n}\right]\right) \\
& \leq \widehat{C}_{2} h^{r-1}\left(\frac{h}{H}\left|u\left(t_{n}\right)\right|_{W_{\infty}^{r}(\tau)}+\left(\frac{H^{r}}{h^{r-1}}+h+\frac{h^{2-\varepsilon}}{H}\right)\left[u ; t_{n}\right]\right) \\
& \leq \widehat{C}_{2} h^{r-1}\left(\frac{h}{H}\left|u\left(t_{n}\right)\right|_{W_{\infty}^{r}(\tau)}+\left(\frac{H^{r}}{h^{r}} h^{\varepsilon}+\frac{h}{H}\right) h^{1-\varepsilon}\left[u ; t_{n}\right]\right) \\
& \leq \widehat{C}_{2} h^{r-1} \widehat{m}\left(\left|u\left(t_{n}\right)\right|_{W_{\infty}^{r}(\tau)}+h^{1-\varepsilon}\left[u ; t_{n}\right]\right),
\end{aligned}
$$

where $\widehat{m}=(H / h)^{r} h^{\varepsilon}+h / H$.

Using this we shall now consider the two alternatives involved in Theorem 2.1.

Alternative 1. In this case we have

$$
\left|u\left(t_{n}\right)\right|_{W_{\infty}^{r}(\tau)} \geq h^{1-\varepsilon}\left[u ; t_{n}\right],
$$

so that by (4.5),

$$
\left|\nabla u\left(t_{n}\right)(x)-G_{H} U^{n}(x)\right| \leq 2 \widehat{C}_{2} h^{r-1} \widehat{m}\left|u\left(t_{n}\right)\right|_{W_{\infty}^{r}(\tau)} .
$$

By Lemma 3.2 and taking $h$ such that $h^{\varepsilon}<\frac{1}{2}$,

$$
h^{r-1}\left(\left|u\left(t_{n}\right)\right|_{W_{\infty}^{r}(\tau)}-\frac{h^{1-\varepsilon}}{2}\left[u ; t_{n}\right]\right) \leq \widehat{C}_{1}\left\|\nabla e^{n}\right\|_{L_{\infty}(\tau)} .
$$

Combining the above estimate with (4.6), we have

$$
\left|\nabla u\left(t_{n}\right)(x)-G_{H} U^{n}(x)\right| \leq 4 \widehat{C}_{1} \widehat{C}_{2} \widehat{m}\left\|\nabla e^{n}\right\|_{L_{\infty}(\tau)} .
$$

This shows (2.10) with $m=4 \widehat{C}_{1} \widehat{C}_{2} \widehat{m}$.

The estimate (2.11) is a simple consequence of the triangle inequality,

$$
\mathcal{E}(\tau) \leq\left|\nabla u\left(t_{n}\right)(x)-G_{H} U^{n}(x)\right|+\left|\nabla e^{n}(x)\right| \leq(1+m)\left\|\nabla e^{n}\right\|_{L_{\infty}(\tau)} .
$$

Also, if $m<1$,

$$
\left\|\nabla e^{n}\right\|_{L_{\infty}(\tau)} \leq\left|\nabla u\left(t_{n}\right)(x)-G_{H} U^{n}(x)\right|+\mathcal{E}(\tau) \leq m\left\|\nabla e^{n}\right\|_{L_{\infty}(\tau)}+\mathcal{E}(\tau),
$$


which gives (2.12).

Alternative 2. In this case, from (4.1) we have

$$
\left\|\nabla e^{n}\right\|_{L_{\infty}(\tau)} \leq 2 \widehat{C} h^{r-\varepsilon}\left[u ; t_{n}\right]
$$

and from (4.5),

$$
\left\|\nabla u\left(t_{n}\right)-G_{H} U^{n}\right\|_{L_{\infty}(\tau)} \leq 2 \widehat{C}_{2} \widehat{m} h^{r-\varepsilon}\left[u ; t_{n}\right] .
$$

By the triangle inequality then,

$$
\mathcal{E}(\tau) \leq\left(m+C_{2}\right) h^{r-\varepsilon}\left[u ; t_{n}\right] .
$$

This shows (2.14), (2.15), and (2.16) with $m=2 \widehat{C}_{2} \widehat{m}$ and $C_{2}=2 \widehat{C}$. Thus the proof of Theorem 2.1 is complete.

\section{NUMERICAL ILlUSTRATIONS}

To illustrate the theoretical results above, we consider a simple one dimensional problem

$$
\begin{aligned}
u_{t}(x, t)-(1+x) u_{x x}(x, t) & =f(x, t), \text { for } 0 \leq x \leq 1, t \geq 0, \\
u_{x}(0, t) & =0, t \geq 0, \\
u_{x}(1, t) & =0, t \geq 0, \\
u(x, 0) & =0,0 \leq x \leq 1 .
\end{aligned}
$$

In all examples the finite elements are quadratic Lagrange elements. The meshes are quasi-uniform but not uniform. In fact, they were constructed starting with a uniform mesh of size $h$ and then perturbing points randomly. Also, in all examples we report at time $t=1$ on the efficiency index, $\mathcal{E}(\tau) /\|\nabla e\|_{L_{\infty}(\tau)}$, where $\tau$ is the element containing $x=1 / 2$. "Patch size" stands for the number of neighboring elements on each side of $\tau$ in the patch $d_{H}$, i.e. patch size 1 means there are three elements in the patch $d_{H}$, patch size 2 means there are five elements and so on.

Below we compare the performances of three different methods.

Method 1: (Local interpolation). Put

$$
G_{H} u_{h}=\frac{\partial}{\partial x} I_{H}^{3} u_{h},
$$

where $I_{H}^{3}: \mathcal{C}\left(d_{H}\right) \rightarrow \mathbb{P}^{3}\left(d_{H}\right)$ is the local Lagrange interpolant onto cubic polynomials on $d_{H}$.

We remark that to avoid phenomena of superconvergence, the interpolation points were taken away from the superconvergent points for the first derivative.

Method 2: (Local $L_{2}$ projection). Put

$$
G_{H} u_{h}=\frac{\partial}{\partial x} P_{H}^{3} u_{h}
$$

where $P_{H}^{3}: L_{2}\left(d_{H}\right) \rightarrow \mathbb{P}^{3}\left(d_{H}\right)$ is the local $L_{2}$ projection onto cubic polynomials on $d_{H}$.

Method 3: (Local $L_{2}$ over-projection). Put

$$
G_{H} u_{h}=\frac{\partial}{\partial x} P_{H}^{4} u_{h}
$$

where $P_{H}^{4}: L_{2}\left(d_{H}\right) \rightarrow \mathbb{P}^{4}\left(d_{H}\right)$ is the local $L_{2}$ projection onto quartic polynomials on $d_{H}$.

After this set-up, we now give our numerical illustrations. 
Example 1. In the first example we take $f$ such that the exact solution is

$$
u(x, t)=t \cos (\pi x) .
$$

For the time discretization, we use the Backward Euler method with uniform time steps. Since the Backward Euler method is exact in this case, the error only depends on the space discretization.

Table 1. ( 40 elements)

\begin{tabular}{|c|c|c|c|}
\hline patch size & Method 1 & Method 2 & Method 3 \\
\hline 1 & 1.2101 & 1.0345 & 1.0320 \\
2 & 0.9390 & 1.0306 & 1.0181 \\
3 & 0.9451 & 1.0525 & 1.0348 \\
4 & 0.9531 & 1.1135 & 1.0838 \\
5 & 1.0404 & 1.2292 & 1.1878 \\
\hline
\end{tabular}

Table 2. (1280 elements)

\begin{tabular}{|c|c|c|c|}
\hline patch size & Method 1 & Method 2 & Method 3 \\
\hline 1 & 1.1641 & 1.0269 & 1.0313 \\
2 & 0.9573 & 1.0149 & 1.0179 \\
3 & 0.9551 & 1.0078 & 1.0076 \\
4 & 0.9540 & 1.0054 & 1.0053 \\
5 & 0.9589 & 1.0037 & 1.0038 \\
\hline
\end{tabular}

As we see from the tables above, the efficiency indices are close to 1 as predicted by the theory.

Example 2. This time we take the exact solution to be

$$
u(x, t)=t^{3} \cos (\pi x) .
$$

For the time discretization we use the Backward Euler method with uniform time steps.

Table 3. (40 space intervals and 160 time steps)

\begin{tabular}{|c|c|c|c|}
\hline patch size & Method 1 & Method 2 & Method 3 \\
\hline 1 & 0.4145 & 0.3684 & 0.3673 \\
2 & 0.3462 & 0.3684 & 0.3654 \\
3 & 0.3478 & 0.3783 & 0.3712 \\
4 & 0.3509 & 0.3978 & 0.3872 \\
5 & 0.3714 & 0.4366 & 0.4182 \\
\hline
\end{tabular}

Table 4. (40 space intervals and 1280 time steps)

\begin{tabular}{|c|c|c|c|}
\hline patch size & Method 1 & Method 2 & Method 3 \\
\hline 1 & 0.9435 & 0.8385 & 0.8361 \\
2 & 0.7880 & 0.8385 & 0.8318 \\
3 & 0.7915 & 0.8610 & 0.8450 \\
4 & 0.7984 & 0.9052 & 0.8813 \\
5 & 0.8449 & 0.9934 & 0.9521 \\
\hline
\end{tabular}


Table 5. (40 space intervals and 10240 time steps)

\begin{tabular}{|c|c|c|c|}
\hline patch size & Method 1 & Method 2 & Method 3 \\
\hline 1 & 1.1233 & 0.9983 & 0.9955 \\
2 & 0.9381 & 0.9983 & 0.9903 \\
3 & 0.9423 & 1.0250 & 1.0060 \\
4 & 0.9505 & 1.0777 & 1.0493 \\
5 & 1.0058 & 1.1826 & 1.1335 \\
\hline
\end{tabular}

As we see from the tables above, these results are in agreement with the theory. The localization effect become evident only when time step is sufficiently small. Thus in Table $3, k=1 / 160$ and $h^{2} \approx 1 / 1600$, hence the time discretization error dominates and we see that the performance of the error estimator is poor. In Table $4, k \approx h^{2}$ and the performance is reasonable. Finally, in Table $5, k \ll h^{2}$ and the performance is very good.

Example 3. Same problem as in Example 2 with the only difference that for the time discretization we now use the Crank-Nicolson method with uniform time steps. Although Theorem 2.1 does not cover the Crank-Nicolson method, standard arguments, (cf. Theorem 2.3 in Thomée, Xu, and Zhang [13]), can be adapted to our problem up to dimension 2. Since the time discretization is of second order, we see good results already with 40 time steps.

Table 6. (40 space intervals and 20 time steps)

\begin{tabular}{|c|c|c|c|}
\hline patch size & Method 1 & Method 2 & Method 3 \\
\hline 1 & 1.0404 & 0.8991 & 0.9070 \\
2 & 0.8310 & 0.8983 & 0.8939 \\
3 & 0.8336 & 0.9157 & 0.9039 \\
4 & 0.8405 & 0.9751 & 0.9527 \\
5 & 0.8905 & 1.0806 & 1.0423 \\
\hline
\end{tabular}

Table 7. (40 space intervals and 40 time steps)

\begin{tabular}{|c|c|c|c|}
\hline patch size & Method 1 & Method 2 & Method 3 \\
\hline 1 & 1.1468 & 0.9910 & 0.9998 \\
2 & 0.9159 & 0.9902 & 0.9853 \\
3 & 0.9188 & 1.0093 & 0.9963 \\
4 & 0.9265 & 1.0749 & 1.0501 \\
5 & 0.9816 & 1.1912 & 1.1489 \\
\hline
\end{tabular}

Example 4. In the last example we take the exact solution

$$
u(x, t)=t^{3} \cos (2 \pi x) .
$$

We use the Backward Euler method with uniform time steps.

Table 8. (40 space intervals and 10240 time steps)

\begin{tabular}{|c|c|c|c|}
\hline patch size & Method 1 & Method 2 & Method 3 \\
\hline 1 & 0.0014 & 0.0025 & 0.0007 \\
2 & 0.0049 & 0.0071 & 0.0005 \\
3 & 0.0097 & 0.0130 & 0.0006 \\
4 & 0.0162 & 0.0237 & 0.0007 \\
5 & 0.0277 & 0.0320 & 0.0010 \\
\hline
\end{tabular}

As we see from the table above, the performance of the error estimator is very poor even for a very small time step. This is in agreement with the theory since 
we are in the case of Alternative 2; indeed,

$$
\frac{\partial^{3} u}{\partial x^{3}}=-(2 \pi)^{3} t^{3} \sin (2 \pi x),
$$

vanishes at $x=1 / 2$.

Acknowledgments: This research was partly supported by an NSF VIGRE Fellowship and U.S. National Science Foundation, under grant DMS 0310539.

\section{REFERENCES}

1. R.E. Bank and J. Xu, Asymptotically exact a posteriori error estimators, part 1: Grids with superconvergence, SIAM J. Numer. Anal., 41 (2003), 2294-2312.

2. R.E. Bank and J. Xu, Asymptotically exact a posteriori error estimators, part 2: General unstructured grids, SIAM J. Numer. Anal., 41 (2003), 2313-2332.

3. P. Brenner, M. Crouzeix, and V. Thomée, Single step methods for inhomogeneous linear differential equations in Banach space, RAIRO Anal. Numer. 16 (1982), 5-26.

4. V. Carey, A posteriori error estimation via recovered gradients, Ph.D. thesis, Cornell University, 2005.

5. S.D. Ė̆del'man and S.D. Ivasišen, Investigation of the Green's matrix for a homogeneous parabolic boundary value problem, Trans. Moscow Math. Soc. 23 (1970), 179-242.

6. A. Hansbo, Nonsmooth data error estimates for damped single step methods for parabolic equations in Banach space, Calcolo, 36 (1999), 75-101.

7. W. Hoffmann, A.H. Schatz, L.B. Wahlbin, and G. Wittum, Asymptotically exact a posteriori estimators for the pointwise gradient error on each element in irregular meshes. Part 1: A smooth problem and globally quasi-uniform meshes, Math. Comp. 70 (2001), 897-909.

8. O. Lakkis and C. Makridakis, Elliptic reconstruction and a posteriori error estimates for fully discrete linear parabolic problems, Math. Comp. Electronically published May 26, 2006.

9. A.H. Schatz, Pointwise error estimates and asymptotic error expansion inequalities for the finite element method on irregular grids: Part 1, Math. Comp. 67 (1998), 877-899.

10. A.H. Schatz, V. Thomée, and L.B. Wahlbin, Stability, analyticity, and almost best approximation in maximum-norm for parabolic finite element equations, Comm. Pure Appl. Math. 51 (1998), 1349-1385.

11. A.H. Schatz and L.B. Wahlbin, Asymptotically exact a posteriori estimators for the pointwise gradient error on each element in irregular meshes. Part II: The piecewise linear case., Math. Comp. 73 (2004), 517-523.

12. V. Thomée, Galerkin Finite Element Methods for Parabolic Problems, Springer Series in Computational Mathematics No.25, Springer-Verlag, Berlin, 1997.

13. V. Thomée, J.-C. Xu, and N.-Y. Zhang, Superconvergence of the gradient in piecewise linear finite-element approximation to a parabolic problem, SIAM J. Numer. Anal., 26 (1989), 553573.

14. S. Ziukas and N.-E. Wiberg, Adaptive procedure with superconvergent patch recovery for linear parabolic problems, Finite element methods, superconvergence, post-processing, and a posteriori estimates, Marcel Dekker, Inc., New York-Basel-Hong Kong, 1998, 303-314.

Department of CAAM, Rice University, Houston, TX 77005

E-mail address: dmitriy@caam.rice.edu

Department of Mathematics, Cornell University, Ithaca, NY 14853

E-mail address: wahlbin@math.cornell.edu 